\title{
Radio-sensitization of SHG44 glioma cells by Aidi injection in vitro
}

\author{
XIAO-TING XU, YAO SONG, SONGBING QIN, LI-LI WANG and JU-YING ZHOU \\ Department of Radiation Oncology, The First Affiliated Hospital Of Soochow University, Suzhou, Jiangsu 215006, P.R. China
}

Received November 30, 2011; Accepted March 1, 2012

DOI: $10.3892 / \mathrm{mmr} .2012 .836$

\begin{abstract}
In this study, we aimed to explore the radio-sensitization of the SHG44 glioma cell line by Aidi injection and the possible mechanisms involved. The growth curve, cloning efficiency and divisional index of the SHG44 cell line were observed. The inhibition ratio was determined by MTT assay, the change in the cell cycle was analyzed by flow cytometry and the expression of cyclin B1 and Weel was detected by western blot analysis. The reproductive activity of the group treated with irradiation (IR) and Aidi injection was suppressed significantly, and the cloning efficiency and divisional index also declined. Aidi injection (15 $\mu \mathrm{g} / \mathrm{ml})$ induced $\mathrm{G} 2 / \mathrm{M}$ phase arrest efficiently in the cell line after $48 \mathrm{~h}$. The expression of cyclin B1 decreased in the group treated with IR and Aidi injection compared with either of those with IR or Aidi injection alone. The expression of Weel increased in the group treated with IR and Aidi injection compared with that in the groups treated with either IR or Aidi injection alone. In conclusion, Aidi injection is effective in radio-sensitization. The possible mechanisms involved may be associated with G2/M phase cell arrest, the downregulation of cyclin B1 and upregulation of Weel expression.
\end{abstract}

\section{Introduction}

Gliomas are one of the most common malignant types of brain tumors. In recent years, the incidence rate of gliomas has shown an increasing tendency worldwide. Surgery and chemoradiotherapy can enhance the curative effect on gliomas to a certain extent. However, concerning treatment, a satisfactory effect has not yet been achieved. Radio-sensitizers play an essential role in the enhancement of the curative effect of radiation. Therefore, it could prove useful to find an effective radio-sensitizer with low toxicity and high safety.

In this study, we investigated the effect of radio-sensitization on the SHG44 glioma cell line by Aidi injection in vitro.

Correspondence to: Dr Ju-Ying Zhou, Department of Radiation Oncology, the First Affiliated Hospital of Soochow University, Suzhou, Jiangsu 215006, P.R. China

E-mail: zhjuying@sohu.com

Key words: SHG44 cell line, Aidi injection, radio-sensitization, $\mathrm{G} 2 / \mathrm{M}$ phase arrest
The possible mechanisms involved were also explored. Our results may provide an experimental basis for the application of the Andi injection in clinical practice.

\section{Materials and methods}

Cell culture and drugs. The SHG44 glioma cell line was cultured in DMEM medium which contained $10 \%$ calf serum in $5 \% \mathrm{CO}_{2}$ at $37^{\circ} \mathrm{C}$. The Aidi injection used in this study, consisting of Radix Ginseng, Astragalus membranaceus Bge, Radix Acanthopanacis Senticosi and Mylabris (0.3 g crude drugs $/ \mathrm{ml}$ ), was a product of GuiZhou YiBai Pharmaceutical Co., Ltd (the manufacturing permit is GUO YAO ZHUN ZI Z52020236).

MTT assay. Cells were cultured for $48 \mathrm{~h}$ and then synchronized at $37^{\circ} \mathrm{C}$. A total amount of $100 \mu \mathrm{l}$ culture medium which contained Aidi injection at the concentrations of 3, 6, 12, 24, 48, 96 and $192 \mu \mathrm{g} / \mathrm{ml}$ were added. The cells were cultured for $48 \mathrm{~h}$. A total amount of $15 \mu \mathrm{l} \mathrm{MTT}$ at $5 \mathrm{mg} / \mathrm{ml}$ was added which then continued to react for $4 \mathrm{~h}$, while $180 \mu \mathrm{l}$ of DMSO were added. After the brown crystalline material dissolved completely, the absorbance (A) of substrate cleavage was monitored at $570 \mathrm{~nm}$ (corrected at $630 \mathrm{~nm}$ ) by a microplate reader. The inhibition ratio was calculated based on the following formula: the inhibition ratio $(\%)=(\mathrm{A}$ vacant control group $-\mathrm{A}$ observed group $) / \mathrm{A}$ vacant control group x $100 \%$.

Clonogenic survival. SHG44 cells were placed in 6-well plates. They were respectively treated with different doses of radiation alone, radiation $24 \mathrm{~h}$ following 9 and $15 \mu \mathrm{g} / \mathrm{ml}$ Aidi injection, and radiation $48 \mathrm{~h}$ following 9 and $15 \mu \mathrm{g} / \mathrm{ml}$ Aidi injection. An accelerator was used for cell radiation (Oncor; Siemens). The cells were then cultured with fresh medium for 14 days. Colonies were fixed with methanol and stained with $1 \%$ crystal violet. Only the colonies containing more than 50 cells were counted. The survival fraction was calculated and analyzed by colony forming assay. The multi-hit singletarget model was used to plot the survival curve. Values of $\mathrm{D}_{0}$ and Dq of the 5 groups were determined and the sensitization enhancement ratios (SERs) by Aidi injection were calculated.

Cell grouping and radiation. The cells were randomized into 4 groups: A, control; B, Aidi injection; $\mathrm{C}$, radiation; and $\mathrm{D}$, Aidi injection and radiation. The cells were cultured at a 
density of $5 \times 10^{4}$ per culture flask for $48 \mathrm{~h}$. Groups B and D were cultured in culture medium containing Aidi injection at $15 \mu \mathrm{g} / \mathrm{ml}$. Groups A and $\mathrm{C}$ were cultured in culture medium without Aidi injection. Groups $\mathrm{C}$ and $\mathrm{D}$ were under $6 \mathrm{Mv} \mathrm{X}$-ray at a tumor dose of $6 \mathrm{~Gy}$.

Flow cytometry. Cells were harvested and centrifuged at $1000 \mathrm{rpm}$ for $6 \mathrm{~min}$. They were washed twice with PBS and then fixed by $75 \%$ alcohol at $4^{\circ} \mathrm{C}$ for $24 \mathrm{~h}$. The cells were washed twice with PBS before analysis. They were resuspended in $400 \mu \mathrm{l}$ PBS and treated with $100 \mu \mathrm{g} / \mathrm{ml}$ RNase A (Roche Diagnostics) and $50 \mu \mathrm{g} / \mathrm{ml}$ propidium iodide (PI) (Sigma). After incubation for $30 \mathrm{~min}$, they were analyzed by Cell Quest software (Becton-Dickinson). Cell cycle distribution was calculated by ModFit LT software.

Western blot analysis. Cells were lysed in solubilizing buffer (1\% Triton X-100, $1 \%$ deoxycholate, $0.1 \%$ SDS, 1 mmol/1 PMSF, and a protease inhibitor cocktail). They were then centrifuged. Samples were separated by $10 \%$ SDS-PAGE and transferred onto PVDF membranes. The membranes were incubated with the primary antibody overnight at $4^{\circ} \mathrm{C}$ (either cyclin $\mathrm{B} 1$ or Wee1). HRP-conjugated rabbit anti-mouse antibody was then applied. Immunoreactive signals were visualized by an ECL detecting system. The optical density value of each protein was normalized to a tubulin level in the same sample. Each experiment was repeated 3 times.

Statistical analysis. Data were expressed as the means \pm SD and analyzed by SPSS 16.0 software. One-way ANOVA was adopted for statistical comparisons among groups. $\mathrm{P}<0.05$ was considered to indicate a statistically significant difference.

\section{Results}

Inhibition ratio. MTT assay revealed that the inhibition ratio of SHG44 cells by Aidi injection was $\mathrm{IC}_{50}=69.2 \mu \mathrm{g} / \mathrm{ml}$. Aidi injection at 9 and $15 \mu \mathrm{g} / \mathrm{ml}$ was used in this study.

Clonogenic survival. The survival rate of the SHG44 cells was decreased by irradiation (IR) alone in a dose-dependent manner. Following Aidi injection, the decrease was even more evident (Fig. 1). After the cells were treated with $15 \mu \mathrm{g} / \mathrm{ml}$ of Aidi injection for $48 \mathrm{~h}$, the SERs reached a maximum of 1.43 (Table I). The survival of SHG44 cells treated with $15 \mu \mathrm{g} / \mathrm{ml}$ of Aidi injection was similar to that of cells in the control group.

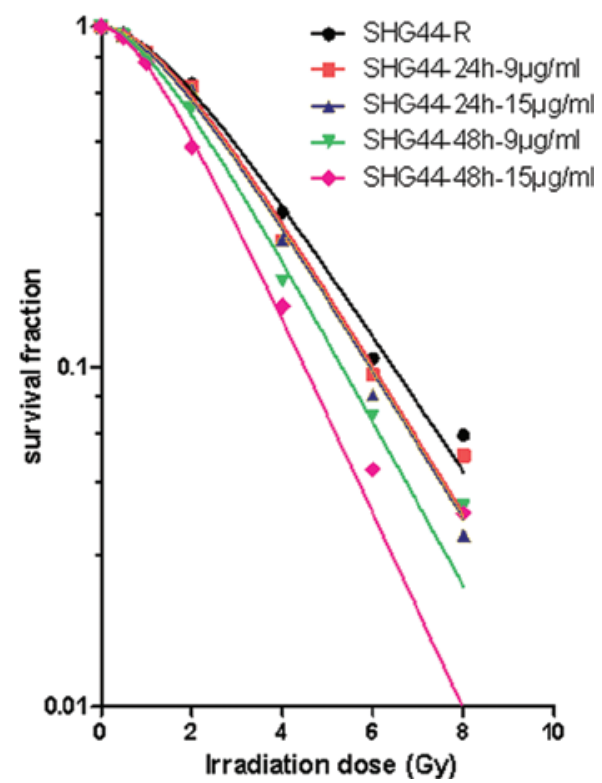

Figure 1. Clonogenic survival fractions of SHG44 glioma cells $24 \mathrm{~h}$ after treatment with irradiation (IR) alone, and 24 and $48 \mathrm{~h}$ after IR combined with 9 and $15 \mu \mathrm{g} / \mathrm{ml}$ of Aidi injection, respectively.

Radiation-induced G2 phase arrest. According to the cell cycle distribution analysis by flow cytometry (Table II and Fig. 2), Aidi increased the proportion of cells in the $\mathrm{G} 2 / \mathrm{M}$ phase in a dose-dependent manner. The proportion of SHG44 cells in G2 phase arrest reached a maximum of $26.86 \pm 1.37 \%$ in the group treated with $15 \mu \mathrm{g} / \mathrm{ml}$ of Aidi injection and $6 \mathrm{~Gy}$ of IR, whereas that in the group treated with $9 \mu \mathrm{g} / \mathrm{ml}$ of Aidi injection and $6 \mathrm{~Gy}$ of IR, as well as that in the group treated with 6 Gy of IR alone was $23.57 \pm 1.76 \%$ and $16.77 \pm 1.10 \%$, respectively. The proportion of cells in G2 phase arrest in the group treated with $15 \mu \mathrm{g} / \mathrm{ml}$ of Aidi injection alone $(19.83 \pm 1.15 \%)$ was much higher compared to that in the controls at the corresponding time-points.

Expression of cyclin BI and Weel. The expression of the cyclin B1 and Wee1 proteins was detected by western blot analysis (Fig. 3). The expression of cyclin B1 in groups B and $\mathrm{C}$ was much higher than that in group $\mathrm{D}$; however, it was much lower in group A. The expression of the Weel protein in groups $\mathrm{B}$ and $\mathrm{C}$ was much lower than that in group $\mathrm{D}$, but much higher in group A.

Table I. Radio-sensitizing parameters of the SHG44 cells treated with Aidi injection.

\begin{tabular}{lccccc}
\hline & $\mathrm{D}_{0}(\mathrm{~Gy})$ & $\mathrm{SF}_{2}(\%)$ & $\mathrm{Dq}(\mathrm{Gy})$ & $\mathrm{N}$ & $\mathrm{SER}\left(\mathrm{D}_{0}\right)$ \\
\hline SHG44-R & 2.14 & 68.0 & 1.58 & 2.09 & - \\
SHG44 (24 h of $9 \mu \mathrm{g} / \mathrm{l})$ & 1.92 & 66.8 & 1.54 & 2.20 & 1.09 \\
SHG44 (24 h of $15 \mu \mathrm{g} / \mathrm{l})$ & 1.99 & 65.5 & 1.44 & 2.08 & 1.08 \\
SHG44 (48 h of $9 \mu \mathrm{g} / \mathrm{l})$ & 1.78 & 57.6 & 1.26 & 2.02 & 1.20 \\
SHG44 (48 h of $15 \mu \mathrm{g} / \mathrm{l})$ & 1.50 & 44.2 & 1.09 & 2.07 & 1.43 \\
\hline
\end{tabular}

SERs, sensitization enhancement ratios. 
Table II. Aidi injection- and radiation-induced G2 phase arrest in SHG44 glioma cells.

\begin{tabular}{|c|c|c|c|c|}
\hline & $0 \mathrm{~Gy}$ & 2 Gy & 4 Gy & $6 \mathrm{~Gy}$ \\
\hline \multicolumn{5}{|l|}{$0 \mu \mathrm{g}$} \\
\hline $\mathrm{G} 1$ & $66.70 \pm 4.23$ & $70.73 \pm 1.32$ & $60.28 \pm 1.18$ & $53.20 \pm 1.41$ \\
\hline G2 & $8.83 \pm 1.21$ & $16.75 \pm 0.41$ & $17.00 \pm 0.50$ & $16.77 \pm 1.10$ \\
\hline $\mathrm{S}$ & $24.50 \pm 3.52$ & $12.45 \pm 1.20$ & $22.72 \pm 1.38$ & $30.03 \pm 1.69$ \\
\hline \multicolumn{5}{|l|}{$9 \mu \mathrm{g}$} \\
\hline G1 & $59.93 \pm 1.90$ & $58.40 \pm 1.10$ & $54.97 \pm 1.37$ & $48.33 \pm 1.15$ \\
\hline $\mathrm{G} 2$ & $9.93 \pm 1.21^{\mathrm{a}}$ & $20.02 \pm 2.03$ & $20.50 \pm 1.97$ & $23.57 \pm 1.76^{c}$ \\
\hline $\mathrm{S}$ & $30.13 \pm 2.65$ & $21.58 \pm 0.93$ & $24.53 \pm 2.91$ & $28.10 \pm 0.62$ \\
\hline \multicolumn{5}{|c|}{$15 \mu \mathrm{g}$} \\
\hline G1 & $58.43 \pm 1.20$ & $58.41 \pm 1.08$ & $58.63 \pm 0.74$ & $48.36 \pm 0.44$ \\
\hline $\mathrm{G} 2$ & $19.83 \pm 1.15^{\mathrm{b}}$ & $19.80 \pm 1.33$ & $20.60 \pm 1.32$ & $26.86 \pm 1.37^{c}$ \\
\hline $\mathrm{S}$ & $21.73 \pm 2.35$ & $21.79 \pm 2.41$ & $20.77 \pm 1.00$ & $24.75 \pm 0.93$ \\
\hline
\end{tabular}

Data are expressed as the means $\pm \mathrm{SD}$ of 3 independent experiments. ${ }^{\mathrm{a}} \mathrm{P}<0.05$ compared with the control group; ${ }^{\mathrm{b}} \mathrm{P}<0.01$ compared with the control group. ${ }^{\mathrm{c}} \mathrm{P}<0.01$ compared with the irradiation alone group.
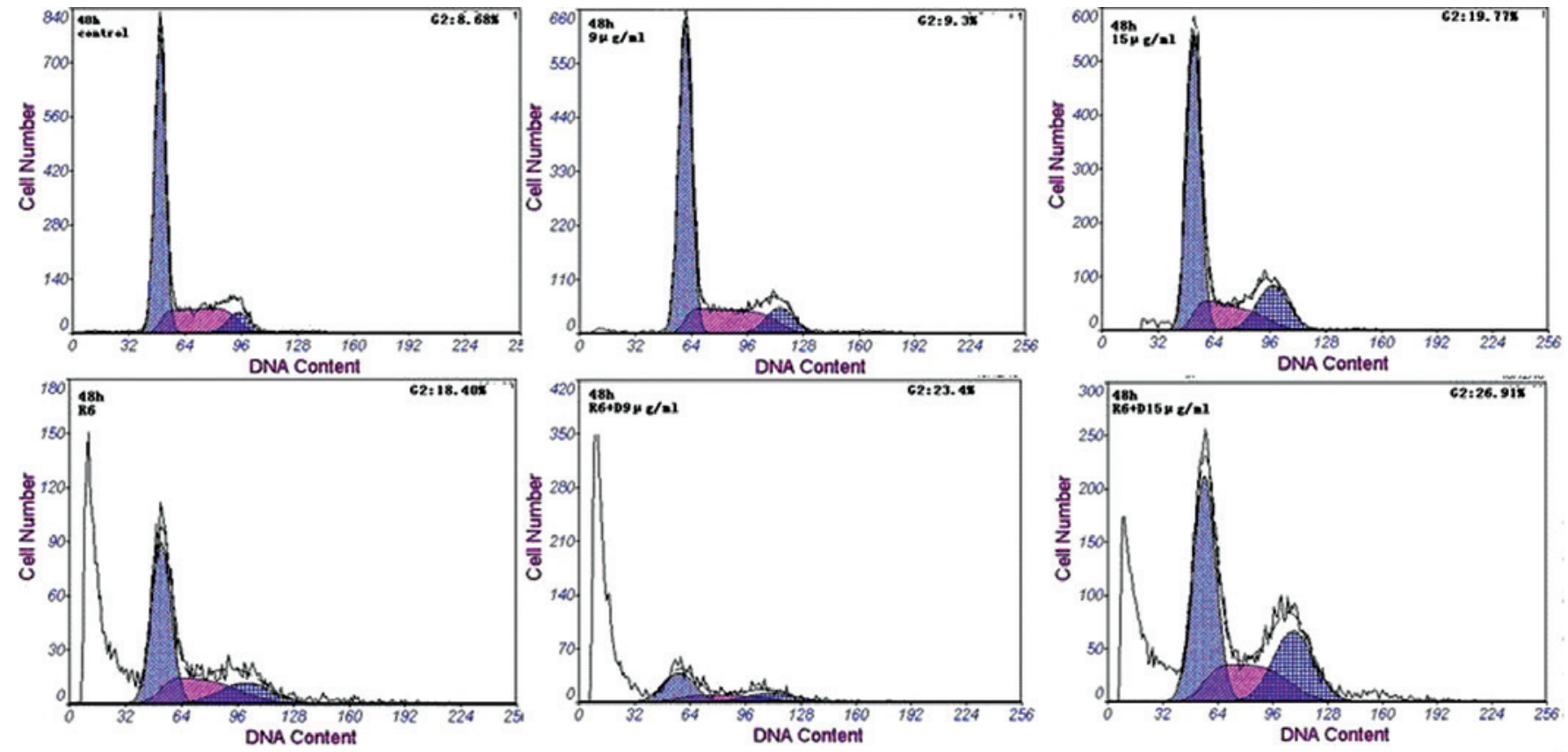

Figure 2. Aidi injection radiation-induced G2 phase arrest in SHG44 glioma cells.

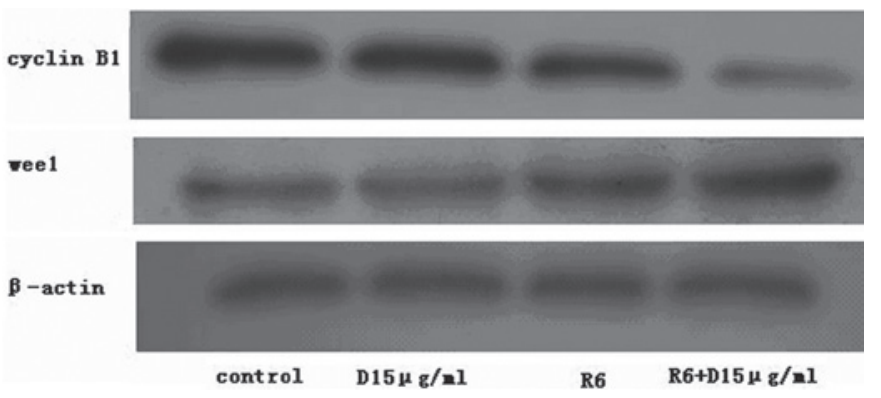

Figure 3. Cyclin B1 and Weel protein expression in SHG44 glioma cells and the effects of irradiation with or without Aidi injection. Data are expressed as the means $\pm \operatorname{SD}$ ( $n=3$ per group).

\section{Discussion}

An increasing amount of evidence has proven that Chinese medicine may play an important role in enhancing the radiochemotherapeutic effect and reducing toxicity, promoting the immune ability of organisms, as well as improving the quality of life and prolonging the survival of patients, etc. Aidi injection is a Chinese medicine extracted from Chinese drugs such as Mylabris (desmethyl-cantharidin) for dissolving node and eliminating toxins and Radix Ginseng, Astragalus membranceys Bge, and Radix Acanthopanacis Senticosi (saponin of ginseng and Astragalus, Acanthopanax polysaccharides, etc.) $(1,2)$. 
Currently, Aidi injection is being used widely in the treatment of lung cancer, esophageal cancer and colorectal carcinoma. It shows a synergistic effect when combined with chemotherapy and radiotherapy. The results from a number of studies have indicated that Aidi injection has a radio-sensitizing effect on CNE-2 nasopharyngeal carcinoma and A549 cells, inducing G2 phase arrest and downregulating BCL-2 expression $(3,4)$. Aidi injection has been shown to enhance the radio-sensitivity of lung cancer cells via the downregulation of the expression of VEGF and SIL-2R in psoriasis (5).

The results from the present study show that Aidi injection improves the anti-tumor effect of IR on SHG44 glioma cells, decreasing the survival rate of the cells. The present study also shows that Aidi injection at the concentration of $15 \mu \mathrm{g} / \mathrm{ml}$ is the optimal radio-sensitizer. It can induce G2/M arrest effectively. Aidi injection induces the radio-sensitization and cycle arrest of SHG44 glioma cells in a dose- and time-dependent manner. Furthermore, the present study suggests that the induction of G2/M arrest in SHG44 glioma cells correlates with the downregulatory effect of Aidi injection on the expression of cyclin B1 and its upregulatory effect on the expression of Wee1.

Cell cycle progression may be controlled via different regulatory pathways. Among these different pathways, a central step is the activation of the cyclin-CDK complex (6), which can be controlled by multiple mechanisms, such as CDK phosphorylation, regulation of cyclin B1 expression and the association of cyclin-CDK complexes with special protein inhibition (7). After Aidi injection or IR treatment, cyclin B1 expression begins to decrease. With the progression of treatment using the Aidi injection and IR, cyclin B1 expression decreases to the minimum. During G2, the dual-specificity kinase, Wee1, is in an active state. It phosphorylates Thr14 and Try15 of CDC2 and keeps the cyclin B1-CDC2 complex in an inactive state. On the contrary, during mitosis, Weel is inactivated and CDC25C activates cyclin B-CDC2 complexes. Therefore, Wee1 and CDC25C play opposite roles in regulating CDC2 expression. To induce G2/M arrest, it is necessary to activate Weel $(8,9)$. Aidi injection or IR can increase Wee1 expression. With the progression of treatment, the expression of Weel is increased to the maximum.

Radiation-induced changes in the blood-brain barrier can aid in the promotion of Aidi injection into the brain to enhance the effect of radio-sensitization on glioma cells $(10,11)$.
Aidi injection is effective as a radio-sensitizing agent for lung cancer (12). It is also effective for glioma. However, as regards its clinical application, further studies are required to further confirm its effectivness.

\section{References}

1. Li HJ, Dong L and Fu SY: Comparative study on treatment of advanced colorectal cancer by Aidi injection combined with FOLFOX4 regimen and FOLFOX4 regimen alone. Integr Tradit West Med 27: 1086-1089, 2007 (In Chinese).

2. Wang Q and Cheng DY: Effect of Aidi injection on peripheral blood expression of Th1/Th2 transcription factors and cytokines in patients with esophageal squamous cell carcinomaduring radiotherapy. Integr Tradit West Med 29: 394-397, 2009 (In Chinese).

3. Ji N, Zhu HX and Ji B: Radiosensitization of Aidi injection on human lung adenocarcinoma cell line A549. J Pract Oncol 25: 60-64, 2010 (In Chinese).

4. Wang JR and Lai RS: The role of Bc1-2 and Bax as well as NF-kB on esophageal squamous cell carcinoma apoptosis induced by Aidi injection. J Liaoning Uni TCM 12: 54-56, 2010.

5. Wang QC and Tian YP: Effect of Aidi injection on SIL-2R and EGFR of non-small cell lung cancer patients following radiotherapy. J Liaoning Univ TCM 10: 68-70, 2008 (In Chinese).

6. Rajewsky FM and Muller R: DNA repair and the cell cycle as targets in cancer therapy. In: The Cancer Handbook. Nature Publishing Group, pp97, 2002

7. Pavletich NP: Mechanisms of cyclin-dependent kinase regulation: structures of Cdks, their cyclin activators, and Cip and INK4 inhibitors. J Mol Biol 287: 821-828, 1999.

8. Parker LL and Piwnica-Worms H: Inactivation of the p34CDC2 cyclin B complex by the human Weel tyrosine kinase. Science 257: 1955-1957, 1992.

9. Graves PR, Yu L, Schwarz JK, et al: The Chk1 protein kinase and the CDC25C regulatory pathways are targets of the anticancer agent UCN-01. J Biol Chem 275: 5600-5605, 2000.

10. Ballabh P, Braun A and Nedergaard M: The blood-brain barrier: an overview: structure regulation and clinical implications. Neurobiol Dis 16: 1-13, 2004.

11. d'Avella D, Cicciarello R, Angileri FF, et al: Radiation-induced blood-brain barrier changes: pathophysiological mechanisms and clinical implications. Acta Neurochir Supp 71: 282-284, 1998.

12. Li Y and Zhu Q: Clinical efficacy of Aidi injection combined with radiotherapy in the treatment of cancer patients. Mod Oncol 13: 672-673, 2005 (In Chinese). 